\title{
Developing Mindful and Targeted Data Visualizations for Diverse Audiences
}

\section{Sanura M. Latham, Charlotte Cherry}

Office of Informatics \& Analytics, Tennessee Department of Health, Nashville, Tennessee, United States

\section{Objective}

Through opioid overdose surveillance data briefs, we aim to focus on creating meaningful targeted reports that incorporate mindful "data points" and visualizations for diverse audiences. Data briefs provide information that is actionable to support decision making across the spectrum of partners involved in responding to Tennessee's opioid epidemic. Additionally, visualizations and reporting of opioid overdose surveillance data create pathways and processes for sharing data and opportunities to collaborate with oth ers' expertise that enrich communication among multi agency collaborators and interdepartmental partners.

\section{Introduction}

Tennessee has experienced an increase of fatal and non-fatal drug overdoses which has been almost entirely driven by the opioid epidemic [1].

Increased awareness by medical professionals, new legislation surrounding prescribing practices, and mandatory use of the state's prescription drug monitoring program has resulted in a decrease of opioid prescriptions and dosages. Paradoxically, emergency department discharges and inpatient hospitalizations due to opioid overdoses have continued to increase. The Tennessee Department of Health, Office of Informatics and Analytics (TDH OIA) has developed visualizations and reports for opioid overdose surveillance data to enhance communication and timely response by health partners. Through opioid overdose surveillance reporting data briefs we aim to focus not on "big data" analytics, but rather meaningfully targeted data briefs that illustrate mindful "data points" and visualizations. These data briefs provide information that is actionable to support decision making across the spectrum of partners involved in responding to Tennessee's opioid epidemic.

\section{Methods}

TDH has partnered with state agencies including the Department of Mental Health and Substance Abuse Services (DMHSAS) and the Tennessee Bureau of Investigation (TBI) as well as internal TDH divisions to foster enhanced opioid response communication. In order to provide a comprehensive yet digestible way to share information we have created two sets of data visualizations that communicate pertinent weekly and monthly opioid overdose trends. A bi-weekly brief contains information from four data sources: Tennessee's Controlled Substance Monitoring Database which is Tennessee's prescription drug monitoring program (PDMP); the Drug Overdose Reporting system which contains non-fatal opioid overdoses captured in hospitals' emergency departments; Vital Records Information System Management which captures fatal drug overdose information; and the Tennessee Incident Based Reporting System which includes opioid and heroin related arrest information. The bi-weekly data brief provides a quick yet inclusive layout of data in an easily consumable manner. A one page front and back layout is divided into four sections, representing each of the four data sources. A nonfatal opioid overdose "counter" displays a year-to-date count of non-fatal opioid overdoses as compared to the previous year.

The monthly report follows a slightly different layout, as the report hones in on data pertaining only to non-fatal opioid overdoses reported from hospital emergency departments. A monthly year-to-date count of non-fatal opioid overdoses and counts of non-fatal opioid overdoses by race and age are included in the report, as well as a visualization depicting the number of non-fatal opioid overdoses by month by opioid class. The monthly report also includes a choropleth map that displays non-fatal opioid overdoses by zip code for the reporting month and a heat map of non-fatal opioid overdoses for cumulative 2018.

Initial feedback from partners about the visualizations included requests to add footnotes for readers to distinguish the data sources and data limitations, as well as requests to provide enhanced contextual information such as year to date counts, previous ye ar counts, and previous month comparisons. Further visualization discussions included requests to add public health regions as a map overlay, identifying metrics that best illuminate "red flags" or upticks in numbers, and assessing whether to display counts or rates for a given data source. 


\section{Results}

Data briefs and reports are disseminated to TDH leadership, the Office of the State Chief Medical Examiner, the Office of General Counsel, TBI, DMHSAS, regional epidemiologists and the newly formed Opioid Overdose Coordinating Office. These data briefs have been proven to be an effective tool for enhanced communication and responding to the opioid crisis. For example, the TDH Commissioner's office requested additional information about a county that was consistently ranking high in non-fatal overdoses as illustrated on a data report. The dissemination of data briefs has also strengthened internal TDH partnerships including linking Viral Hepatitis and HIV programs with OIA to develop visualizations that incorporate HCV and HIV data (Hepatitis C Virus; Human Immunodeficiency Virus) with opioid overdose data to better understand and elucidate the syndemic of opioid overdose, HCV, and HIV in Tennessee.

The dissemination of data briefs and reports has also been an effective tool for responding to the opioid crisis with our interagency partner, DMHSAS. A DMHSAS team utilized the data briefs to inform how and where to expand an overdose recovery navigator program within the state - a program that provides individuals who have recently overdosed (and are still in the emergency room) with information for treatment and recovery resources. Current work, also in partnership with DMHSAS, has been the creation of a data brief specifically on the topic of harm reduction. The data brief will include a map that shows areas of naloxone distribution to law enforcement agencies throughout the state, as well as a map that illustrates naloxone administration locations by law enforcement officers in the field. Additionally, information on locations of syringe exchange programs will be included in the brief. Members from DMHSAS have provided feedback that they anticipate using the harm reduction brief to assess which remaining law enforcement facilities have not yet on-boarded to receive naloxone, to pin point areas where additional distributions of naloxone might be needed, and identify where supplemental naloxone administration trainings for, either law enforcement or the community, might be targeted.

\section{Conclusions}

Visualizations have illuminated patterns and "red flags" in geographic areas that have helped guide decision makers in making data-driven decisions about opioid response. Visualizations and reporting of opioid overdose surveillance data has also importantly enriched communication among multi agency collaborators and interdepartmental partners that, until now, have been largely siloed. Pathways and processes for sharing data and opportunities to collaborate with others' expertise have been strengthened by the dissemination of targeted mindful "data point" briefs over large scale "big data" analytics.

\section{Acknowledgement}

The authors thank their partnership with the Tennessee Department of Mental Health and Substance Abuse Services and the Tennessee Bureau of Investigation. Grant support comes from the US Department of Justice, Office of Justice Programs, Bureau of Justice Assistance, Harold Rogers Prescription Drug Monitoring Program Grant \#2016PM-BX-K002.

\section{References}

1. Office of Informatics and Analytics, Tennessee Department of Health. Prescription drug overdose program 2018 report: understanding and responding to the opioid epidemic in Tennessee using mortality, morbidity, and prescription data [Internet]. Tennessee Department of Health, 2018. Available from: https://www.tn.gov/content/dam/tn/health/documents/pdo/PDO_2018_Report_02.06.18.pdf. 